0

\title{
In vivo imaging of the GnRH pulse generator reveals a temporal order of neuronal activation and synchronization during each pulse
}

\author{
Aleisha M. Moore*1, Lique M. Coolen ${ }^{1}$ and Michael N. Lehman ${ }^{1}$ \\ ${ }^{1}$ Brain Health Research Institute, Kent State University, Kent, OH; Department of Biological \\ Sciences, Kent State University, Kent, $\mathrm{OH}$.
}

*Corresponding author: Aleisha M. Moore. Email: amoor149@kent.edu

Keywords: Fertility, luteinizing hormone, KNDy, kisspeptin, pulses

\section{Abstract}

A

A hypothalamic pulse generator located in the arcuate nucleus controls episodic release of gonadotropin-releasing hormone $(\mathrm{GnRH})$ and luteinizing hormone $(\mathrm{LH})$ and is essential for reproduction. Recent evidence suggests this generator is comprised of arcuate "KNDy" cells, the abbreviation based on co-expression of kisspeptin, neurokinin B, and dynorphin. However, direct visual evidence of KNDy neuron activity at a single-cell level during a pulse is lacking. Here, we use in vivo calcium imaging in freely moving female mice to show that individual KNDy neurons are synchronously activated in an episodic manner, and these synchronized episodes always precede LH pulses. Furthermore, synchronization among KNDy cells occurs in a temporal order, with some subsets of KNDy cells serving as "leaders" and others as "followers" during each synchronized episode. These results reveal an unsuspected temporal organization of activation and synchronization within the GnRH pulse generator, suggesting that different subsets of KNDy neurons are activated at pulse onset than afterward during maintenance and eventual termination of each pulse. Further studies to distinguish KNDy "leader" from "follower" cells is likely to have important clinical significance, since regulation of pulsatile $\mathrm{GnRH}$ secretion is essential for normal reproduction and disrupted in pathological conditions such as polycystic ovary syndrome and hypothalamic amenorrhea. 
Introduction

Reproduction in mammals depends on a hypothalamic pulse generator that regulates the

32 episodic release of gonadotropin-releasing hormone $(\mathrm{GnRH})$ from the hypothalamus $(1,2)$.

33 Regulation of the frequency and amplitude of $\mathrm{GnRH}$ pulses, and, in turn, that of the gonadotropins

34 luteinizing hormone ( $\mathrm{LH})$ and follicle-stimulating hormone (FSH) from the anterior pituitary gland,

35 is essential to regulate steroid hormone production and gamete development at the gonads.

36 Although the first observations of the pulsatile nature of $\mathrm{GnRH}$ and $\mathrm{LH}$ release were made in the

37 1970s, until recently the precise location and cellular identity of the neural pulse generator

38 responsible for episodic $\mathrm{GnRH}$ release remained a major unanswered question.

The 2003 discovery that mutations in the gene encoding the kisspeptin receptor ( $G$ proteincoupled protein 54 (GPR54)) result in hypogonadotropic hypogonadism delivered compelling

41 evidence that kisspeptin-positive cells in the brain are required for maintaining $\mathrm{GnRH}$ release (3,

42 4). Subsequent studies testing the role of kisspeptin in animal models confirmed activation of GnRH

43 neurons via GPR54 to potently stimulate $\mathrm{GnRH}$ and $\mathrm{LH}$ release $(5,6)$. Multi-labeling experiments

44 in sheep later revealed that kisspeptin cells in the arcuate nucleus of the hypothalamus (ARC) also

45 co-expressed two other important mediators of GnRH release; the tachykinin neurokinin B (NKB)

46 and the endogenous opioid peptide (EOP) dynorphin (7); as an abbreviation, these cells were

47 termed KNDy (Kisspeptin/Neurokinin B/Dynorphin) neurons. Colocalization of the three KNDy

48 peptides was subsequently demonstrated in the mouse, rat, cow, goat and non-human primate (8-

49 12). Anatomical characterization of KNDy neurons revealed reciprocal connections and the

50 expression of postsynaptic receptors for NKB and dynorphin, indicating KNDy cells form an

51 interconnected population potentially capable of synchronization $(9,13-16)$. These characteristics

52 provided the basis for the "KNDy hypothesis" of GnRH pulse generation, in which NKB acts as the

53 signal responsible for pulse onset by triggering activation of reciprocally connected KNDy neurons

54 and driving the kisspeptin-mediated secretion of $\operatorname{GnRH}(13,17-20)$. In support of this, bilateral 
55 infusions of NKB or dynorphin antagonists into the mediobasal hypothalamus (MBH) enhances and

56 suppresses LH pulsatile release, respectively, in sheep and goats (12, 21). Further, the conditional

57 inhibition and brief activation of kisspeptin-expressing cells in the ARC using optogenetic tools in 58 mice suppresses and elicits LH pulses, respectively $(22,23)$. Finally, in vivo measurement of KNDy

59 neuron population activity using GCaMP6 fiber photometry in awake and freely moving mice

60 revealed transient increases in intracellular calcium by KNDy neurons before an LH pulse,

61 indicative of episodic activity within the KNDy neuron population that drives pulsatile LH release

62 (22). However, as these studies either manipulate or record the activity of large proportions of the

63 KNDy population, they do not identify individual cells which are responsible for $\mathrm{GnRH} / \mathrm{LH}$ pulse

64 generation, or whether those cells are activated homogeneously during an individual pulse. To

65 address these questions, we conducted in vivo calcium imaging using miniature microscopy in

66 order to visualize KNDy cell activity at single-cell level in freely behaving mice, and, combined this

67 with serial blood sampling to examine the pattern of activation of KNDy cells during an LH pulse.

68 Using this approach, we showed that synchronized activation of KNDy cells always precedes an

69 individual LH pulse. Surprisingly, we also found that not all KNDy cells are synchronized

70 simultaneously during a pulse; rather KNDy cells are recruited in a recurring temporal order

71 comprised of distinct subpopulations of "leader" cells, which activate and reach peak activity at the

72 onset of synchronized episodes, and "follower cells", which reach peak activity during either the

73 maintenance or termination phase of the episode. These results provide an important missing piece

74 in the "KNDy" hypothesis of pulse generation, showing that synchronized activity of individual KNDy

75 neurons precedes each pulse, as well as revealing an unexpected temporal complexity in the

76 cellular organization of this neural pulse generator.

77

78

79

80

81

82

\section{Results}

\section{Confirmation of GCaMP6 expression in ARC kisspeptin cells}


The viral vector used in this study (AAV9-CAG-Flex-GCaMP6s) contains the genetically encoded calcium sensor MP6s (GCaMP6s) and has been previously characterized as highly specific and selective to transfection of kisspeptin cells when injected into the ARC of Kiss1-Cre mice (22). The viral titer was diluted in this study to elongate the experimental timeline without

87 toxicity to cells due to long-term transfection. To confirm selectivity of the viral vector to ARC

88 kisspeptin cells, we unilaterally injected the Cre-dependent viral vector into the ARC of Kiss1Cre/tdTomato reporter mice (Figure $1 \mathrm{~A}$ ). We show here that $98.4 \pm 0.5 \%$ of GCaMP6s-expressing cells were colocalized with tdTomato-expressing ARC kisspeptin cells, indicating, as in previous reports, the AAV is highly specific to Cre-positive cells (Figure $1 \mathrm{~B}, \mathrm{n}=3$ ). Conversely, $70.5 \pm 8.1 \%$ of tdTomato-expressing kisspeptin cells expressed GCaMP6s, showing over two-thirds of the KNDy population express the transgene (Figure $1 \mathrm{C}, \mathrm{n}=3$ ). No GCaMP6s-positive cells were detected in wild-type mice, consistent with Cre being required for transfection $(n=3)$.

The KNDy population at a single-cell level exhibits synchronized increases in intracellular calcium

For in vivo calcium imaging experiments, Kiss1-Cre female mice were bilaterally ovariectomized (OVX) to remove inhibition of LH pulse generation by gonadal steroid hormone negative feedback and underwent Cre-dependent AAV transfection of ARC kisspeptin (KNDy) cells with GCaMP6s. To enable optical access to GCaMP6s-expressing KNDy cells, a 500 $\mu \mathrm{m}$ diameter,

$1028.4 \mathrm{~mm}$ gradient index refractive (GRIN) lens was positioned directly above the ARC and secured 103 via an integrated baseplate. After five weeks of daily handling and habituation, unanesthetized and 104 freely moving OVX Kiss1-GCaMP6s females underwent 60-minute sessions in which calcium 105 fluorescent signal was visualized and recorded at $10 \mathrm{hz}$ via attachment of a single-photon 106 miniaturized microscope (Inscopix Inc, nVoke) to the integrated baseplate, while tail-tip blood 107 samples were collected every 3-4 minutes for LH pulse detection ( $n=5$, Movie S1A, Figure 2A-C). Histological examination at the completion of recording in perfusion-fixed tissue revealed that the 
109 majority of cells recorded were located within the middle region of the ARC. Fluorescent traces 110 were extracted and analyzed from an average of $13 \pm 1.6$ cells per animal using the principal 111 component analysis (PCA)-individual component analysis (ICA) cell detection algorithm (Movie 112 S1B-C, Figure 2D). The average reduction in baseline fluorescence during the first and final 100 113 seconds of baseline activity was $1.8 \pm 1.4 \%$, suggesting little to no photobleaching of cells occurred 114 within the 60-minute recording period.

Visualization of KNDy neurons at the single-cell level revealed episodes of elevated calcium fluorescence across the recording period with an interval of $4.1 \pm 0.7 \mathrm{~min}$ (Figure 2E). No observations of reduced KNDy cell activity were recorded. The average increase in fluorescent 118 intensity relative to resting fluorescent intensity (DF/F) during episodes of activity in KNDy cells 119 across animals was $263.98 \pm 63.7 \%$, but ranged from $117.7 \%$ to $1211.9 \%$ between individual cells. 120 To normalize for differences in DF/F between cells, analysis was henceforth conducted on z-scored 121 cell traces, which was generated by calculating the standard deviation of each recorded timepoint 122 from the mean fluorescence of the cell. correlation between individual cells at baseline activity, there was a strong and significant increase in correlation between cells when the average activity of the population was above baseline (Figure

$1263 \mathrm{~A}-\mathrm{B}, \mathrm{p}<0.05)$. In support of this high correlation during episodic activity, we detected that the 127 majority of episodes $(75.2 \pm 5.7 \%)$ were the result of an increase in calcium signal by all recorded 128 KNDy cells, representative of synchronized activation of the KNDy neuronal network and referred 129 henceforth as synchronized episodes (SEs). In addition to SEs, episodes were also detected in 130 which smaller percentages of the recorded KNDy cells (between 7.1-71.4\%) showed episodes of 131 elevated fluorescence, indicative of heterogenous KNDy cell activity (Figure 3C). To compare 132 whether full versus partial recruitment of the KNDy population affected the strength and duration of 133 network activation; the amplitude at the peak of an episode, the width of the episode at half 134 amplitude, and the time that calcium fluorescence was above baseline was compared between 
135 SEs and episodes with only subpopulations of cells displaying activation. The average amplitude 136 at the peak of cell activation was dramatically and significantly higher in KNDy cells during a SE 137 compared to episodes in which only partial activation of the KNDy neuronal population was 138 achieved (Figure 3D-E, $\mathrm{p}<0.05$ ). Surprisingly, a positive linear relationship was not detected 139 between the percentage of cells activated versus the amplitude at episode peak $\left(R^{2}=0.0251\right)$. 140 Instead, except for one episode (marked \# in Figure 3D), the average amplitude of the episode 141 peak remained low unless all cells were synchronously activated. The width (s) at half amplitude, 142 a measure of the duration of the episode, was also significantly higher during SEs compared to 143 episodes where subpopulations were activated ( $p<0.05$, Figure $3 F)$. The time for cells to reach 144 peak amplitude from baseline was not significantly different between SE and episodes with less 145 than $100 \%$ of cells active (Figure 3G). However, the time from peak amplitude to return to baseline 146 was significantly higher during SE's compared to episodes with less than $100 \%$ of cells active 147 (Figure $3 \mathrm{H}, \mathrm{p}<0.05)$. Overall, the total time above baseline was significantly higher during SE's 148 compared to episodes with less than $100 \%$ of the recorded cells showing activity (Figure $3 \mathrm{l}$, $149 \mathrm{p}<0.05)$. Together, these data indicate that recruitment of all cells during a SE result in KNDy cell 150 activity with both higher amplitude and longer duration.

152 KNDy neurons display a temporal order of activation and peak amplitude during a 153 synchronized episode

The order in which cells 1) displayed fluorescent signal elevated above baseline, termed 155 here as the time of cell activation (Figure $4 \mathrm{Ai}, \mathrm{Bi}$ ) and 2) reached peak amplitude (Figure 4 Aii, Bii) 156 was analyzed across all SEs recorded from each animal. Across the average of $11.2 \pm 0.9$ SEs 157 recorded in 60-minutes, $45.9 \pm 4.1 \%$ of recorded KNDy cells displayed at least one instance of 158 activating first during an SE. Cells that were activating first did so on multiple SEs, on average, 2.2 $159 \pm 0.3$ times out of the $11.2 \pm 0.9$ SEs (or $19.8 \pm 0.9 \%$ of SEs) (Figure 4 C). Likewise, we found that $16036.6 \pm 6.1 \%$ of recorded KNDy cells displayed at least one instance of reaching peak amplitude 
161 first. Cells that were peaking first would do so on an average of $3.1 \pm 0.5$ out of $11.2 \pm 0.9$ episodes

162 (or $26.72 \pm 3.2 \%$ of SEs) (Figure 4C). These data indicate the presence of a potential "leader cell"

163 subpopulation, in which cells take turns to repeatedly activate or reach peak amplitude first. We

164 next aimed to assess the order in which cells in this subgroup activate and reach peak amplitude

165 when not leading the generation of an SE by activating or peaking first. To achieve this, we

166 quantified the cumulative percentage of the cell population that was recruited (e.g. the percentage

167 of KNDy cells that activated or peaked first or second; first, second or third; etc.) (Figure 4D). Up

168 until the point that $33.3 \%$ of the total KNDy cell population had activated or reached peak amplitude,

169 the size of the KNDy neuron population recruited was not significantly different from the size of the

170 cell population that activated or peaked first. This indicates that when cells with a recorded instance

171 of activating or peaking first are not leading an episode, they will still activate or peak early within

172 an SE prior to two-thirds of the remaining population. An example of this can be seen in Figure 4

173 Bii where KNDy cells that peak first will, on alternate episodes, reach peak amplitude either second,

174 third or fourth. Finally, we determined that only $24.9 \pm 3.6 \%$ and $26.2 \pm 5.3 \%$ of the recorded KNDy

175 population exhibited at least one instance where they were last to activate or peak during the hour-

176 long recording window, respectively (Figure 4C). Therefore, cells that were last to activate or peak

177 in an SE did so $5.1 \pm 0.9$ and $5.4 \pm 1.3$ times out of $11.2 \pm 0.9$ episodes during the 60 -minute

178 recording. Only $6.2 \pm 5.3 \%$ and $4.5 \pm 2.9 \%$ of cells that activated or peaked first at least once

179 during the recording period also displayed an instance of activating or peaking last. Together, these

180 results suggest that KNDy cells are segregated into subpopulations of "leader" cells that either

181 activate or peak first, and "follower" cells that either maintain or terminate the episode.

\section{Synchronized episodes are initiated by a slowly activating population of leader cells}

Although the percentage of KNDy cells capable of activating first was not significantly different to the population of cells that peak first (Figure 4C-D, $p>0.05$ ), only $9.1 \pm 2.6 \%$ of SEs exhibited a cell that both activated first and reached peak amplitude first. Visual inspection of cell 
187 traces noted two patterns of activity that occurred between the baseline and peak of episodes. The

188 first pattern would exhibit a rapid increase in fluorescence from baseline to peak, followed by a 189 slower reduction in fluorescence to baseline (Figure 4E-F, blue line). The second pattern would 190 exhibit a slower increase in fluorescence before reaching the peak of the episode (Figure 4E-F, red 191 line). These two patterns were not observed within distinct cell subpopulations. Instead, cells could 192 exhibit either pattern in different SEs. In line with these observations, cells that were first to activate 193 during an SE took significantly longer, by $6.9 \pm 1.5$ seconds, to reach peak amplitude compared to 194 the cells that reached peak amplitude first (Figure 4G, $p<0.05$.).

\section{LH pulses are preceded by synchronized episodes of KNDy cell activity}

Serial blood samples were collected from mice throughout the 60-minute recording periods to correlate KNDy population activity with LH pulsatile release (Figure $5 \mathrm{~A}$ ). As expected in OVX female mice, a rapid frequency of $7.4 \pm 0.9 \mathrm{LH}$ pulses per hour was recorded. All LH pulses were

200 preceded by KNDy neuron population activation, with an average interval of $3.1 \pm 0.3$ minutes

201 lapsing between the peak amplitude of an LH pulse and the peak of the immediately preceding fluorescent episode. Although LH pulses faithfully followed KNDy neuron activation, only $49 \pm 7.8 \%$ of all detected KNDy neuron activation episodes were followed by an LH pulse. Notably, LH pulses primarily followed the SEs, as $59.6 \pm 6.1 \%$ of SEs (with $100 \%$ of cells activated) were followed by an LH pulse, while only one episode in which less than $100 \%$ of cells were activated $(3.3 \pm 3.3 \%$ of subpopulation episodes) elicited LH pulsatile release (Figure 5B). In addition, although the average interval of time between the peak of SEs (Inter-SE-Interval, ISI) across animals was 5.3 \pm 0.4 min, we compared LH output within a variety of ISIs. The majority of SE's had an ISI of over 4 minutes, although two animals exhibited episodes in which the ISI was more rapid (Figure 5C). When analyzing LH pulsatile release after KNDy SEs at varying ISIs, we found that most SEs with an ISI of over 5.5 minutes elicited an LH pulse (Figure 5D). When the ISI dropped beneath 5.5 minutes, the incidence of LH pulse generation was significantly reduced, indicating that the 
213 detection of an LH pulse depends on the ISI (Figure 5D, Figure 5Fi-iii). Effects of ISI on LH pulse

214 amplitude were also noted. The amplitude of LH release following SEs with an ISI of 4-5.5min was

215 not significantly different compared to episodes with an ISI of over 5.5 minutes (Figure $5 E, p>0.05$ ).

216 However, in the two animals that exhibited SEs with an ISI of under 4 minutes and some LH pulses

217 were detected (Figure 5D), the amplitude of the LH pulses was markedly lower compared to pulses

218 following SEs with a larger ISI (Figure 5E). Finally, no significant difference was detected in the

219 amplitude of SEs that were immediately followed by an LH pulse versus the amplitude of SEs that

220 were not followed by an LH pulse ( $4.31 \pm 0.2$ vs $4.48 \pm 0.4$ Z-scored DF/F, $p=0.7)$. Together, these

221 data demonstrate that synchronized activation of the KNDy population precedes LH pulsatile

222 release, but this relationship is not perfectly correlated when KNDy SEs are generated at high

223 frequency.

224

225

226

227

228

229

230

231

232

233

234

235

\section{Discussion}

Using in vivo calcium imaging at single-cell resolution in freely behaving female mice, we demonstrate here that ARC KNDy cells, proposed as the GnRH pulse generator, show synchronous activation of all recorded cells prior to pulsatile LH release. Strikingly, we identified that the order in which cells fire during a synchronized episode is temporally organized, revealing subsets of "leaders" and "followers" that may be critical for the initiation, maintenance, and termination of $\mathrm{GnRH}$ pulse generation. Together, these data provide critical support that KNDy cells are the hypothalamic $\mathrm{GnRH} / \mathrm{LH}$ pulse generator and novel evidence for subsets within the cell population that may dictate the initiation of activity within this reciprocally connected population.

Sixty-minute recordings of GCaMP6s fluorescent signal from the KNDy population at single cell resolution revealed that activation of all recorded cells occurred in approximately $75 \%$ of episodic events. This data supports anatomical $(14,16,24)$ and in vitro electrophysiological data (25) which suggest KNDy cells form an interconnected network capable of synchronization. It further extends these observations by demonstrating that highly correlated synchronized activity 
241 occurs amongst all KNDy cells detected during an episode and always precedes an LH pulse. In 242 most animals, we also detected episodes in which less than $100 \%$ of the cell population displayed 243 activity. In these instances, the mean amplitude of the calcium signal across cells was significantly 244 lower and not followed by LH release. Although this may indicate that complete synchronization of 245 the KNDy population is necessary to drive LH secretion, this is not necessarily supported by 246 previous reports. For instance, bilateral lesioning of KNDy cells can disrupt LH release (26-28), but 247 the unilateral suppression of KNDy neurons using inhibitory optogenetics is insufficient to suppress 248 LH pulsatile release (22). Similarly, the knockdown of approximately $30 \%$ of the KNDy cell 249 population does not have an appreciable effect on LH pulse generation besides a slight reduction 250 in LH pulse frequency (29). Combined with our results, this suggests that, in situ, all KNDy cells 251 may participate in pulse generation, but not all cells may necessarily be required. Potentially, the 252 larger amplitude recorded during endogenous synchronized episodes may be a critical factor for 253 LH release. Currently, the role of smaller amplitude episodic events from subsets of the KNDy 254 population is unknown. It is possible that these episodes represent failed attempts to release the 255 necessary signal, such as NKB, believed to be a start signal for each pulse $(9,12,21,30)$, that 256 drives a full synchronized episode and LH release.

Another consideration is that our data was collected from cells primarily in the middle division of the ARC. It is possible that KNDy cells in more rostral or more caudal divisions may not 259 show the same synchronized activity before an LH pulse. For instance, previous attempts to 260 suppress LH pulsatile release using inhibitory optogenetics was successful in the middle and 261 caudal ARC, but not the rostral portion of the nucleus (22). Further, projections from the rostral half 262 of the arcuate differ to projections from more caudal neurons (31). These data suggest that 263 although we recorded from cells displaying synchronized activity consistent with pulse generation, 264 there may be anatomically and functionally distinct subpopulations in other ARC regions that do 265 not demonstrate the episodic activity prior to LH release and instead are related to other functions 266 of KNDy cells, such as their role in the modulation of metabolic responses and/or stress (32-35). 
268 synchronized episode (SE). Analysis across multiple SEs within 60-minute recording periods from

269 each animal revealed that a subpopulation of the recorded cells peaked first at least once. When

270 not first, this subpopulation was still temporally restricted to reach peak amplitude early within an

271 episode. Similarly, cells that peaked during either the mid-way or endpoint of the event were

272 restricted to this timepoint across all SEs studied. This suggests the possibility that the KNDy

273 population contain a subset of "leader" cells capable of driving the initiation phase of an episode

274 and "follower" cells that only fire during the maintenance and termination phase. Surprisingly, cells

275 which were first to exhibit an increase in activity above baseline (activate) at the beginning of an

276 episode were not the first to reach peak amplitude. Analysis of these two potential subpopulations

277 of leader cells, cells first to activate and cells first to peak, confirmed that cells first to activate

278 exhibited a slower 'shoulder' period between baseline and peak amplitude, whereas cells that

279 peaked first demonstrated a more rapid increase in calcium activity until peak amplitude was

280 reached. Potentially, these distinct patterns of activity may indicate a model in which "activating"

281 leader cells undergo a slow increase in activity towards a threshold that elicits the release of NKB

282 onto neighboring KNDy cells. NKB release may then propagate the activation of reciprocally

283 connected KNDy cells to reach peak amplitude, as illustrated in Figure 6. The initial source of

284 excitation for activating leader cells is currently unknown. It is worth noting, the identification of

285 leader cells within synchronized cell populations that drive episodic pituitary hormone output has

286 remained a major unresolved issue in multiple neuroendocrine systems. For instance, oxytocin

287 neurons of the hypothalamus display synchronized bursts of firing that lead to pulses of oxytocin

288 release from the posterior pituitary gland $(36,37)$. However, attempts using techniques such as

289 paired electrophysiological recordings have been unable to determine leader and follower

290 subpopulations (38, 39). Results from our data and from future studies of the KNDy pulse generator

291 may therefore provide insight on the generation of SEs for other neuroendocrine populations as

292 well. 
294 activation of the KNDy cell population always occurred preceding individual LH pulses. Surprisingly,

295 and unlike reports using fiber photometry in male mice and intact female mice (22, 40, 41), we also

296 detected SEs that were not followed by an LH pulse. Further analysis revealed that the vast majority

297 of SEs with an interval between the SE peaks (Inter-SE-Interval, ISI) of over 5.5 minutes generated

298 a detectable LH pulse, whereas SEs with a shorter ISI weakened this relationship. Prior fiber 299 photometry recordings detected near-perfect correlation between LH pulsatile release and 300 preceding KNDy population activity in gonadectomized males with an interval ranging between 4.5 301 and 20.7 minutes (22). However, our observation that rapid SEs were not followed by LH release 302 may have two explanations. First, an LH pulse with an ISI below 5.5 minutes will be difficult to 303 capture using a blood sampling interval of 3 minutes. Here, in ovariectomized female mice, SEs 304 displayed an average ISI of 5.3 minutes and a range between 3 and 9 minutes. The surprisingly 305 fast pace of the $\mathrm{GnRH}$ pulse generator in this study may result from long-term ovariectomy which, 306 in these studies, was typically a 6-8-week period before recording. Currently, we are unable to test 307 whether a brief pulse of LH follows SEs with an interval of under 4 minutes, as collection of mouse 308 tail blood samples more frequently than every 3 minutes in freely moving mice is currently not 309 technically feasible over the length of time required for multiple SEs to occur. Alternatively, high 310 frequency $\mathrm{GnRH}$ pulses may desensitize the pituitary to $\mathrm{GnRH}$, leading to reduced or absent $\mathrm{LH}$ 311 secretion. This is supported by historical pulse generation studies in OVX rhesus monkeys which 312 demonstrated that using exogenous delivery of $\mathrm{GnRH}$ to increase the physiological frequency of $313 \mathrm{GnRH}$ stimulation of the pituitary gland from one pulse an hour to two, three and five pulses an 314 hour resulted in a gradual decline in LH output (42). Similar results have been observed in perifused 315 sheep pituitary cells, in which exogenous delivery of GnRH pulses more rapidly than every 16 316 minutes also reduced $\mathrm{LH}$ release (43). 
320 to peak? Do manipulations of either "leader" or "follower" cells result in altered profiles of GnRH 321 release and downstream effects on pituitary gonadotropin secretion? Given the fundamental role

322 that the $\mathrm{GnRH}$ pulse generator plays in control of reproduction, identification of the mechanisms

323 responsible for temporal ordering of KNDy cells synchronization during a pulse may have important 324 translational relevance for understanding reproductive disease as well as normal function. In the 325 clinic, LH pulse frequency outside the physiological norm leads to significant deficits in reproductive 326 capacity in both male and female patients, manifesting in disorders such as hypothalamic 327 amenorrhea (44) and polycystic ovary syndrome (45). KNDy peptide antagonists have already 328 been intensely studied as therapeutic tools in the treatment of these and other diseases (46-48). 329 Further characterization of leader versus follower cells, and the signaling pathways controlling their 330 synchronization, may provide a basis for development of new therapeutics to either trigger or inhibit 331 KNDy pulses and novel approaches to the control of reproduction.

\section{Materials and Methods}

Animals.

All mice were bred and housed in the Kent State University animal facility on a 12-hour light/dark cycle and given access to food and water ad libitum prior to calcium imaging experiments. Experimental procedures in mice were conducted from 50 days of age. All experimental protocols and procedures were approved by Kent State University Institutional Animal Care and Use Committee under protocol 484 LC 19-08 and conform to guidelines outlined by the United States National Institutes of Health for animal research. Heterozygous Kiss1-Cre mice, in which Crerecombinase expression is driven by Kiss1 regulatory elements (49) (Breeding pairs kindly donated by Dr. Carol Elias, JAX mice, stock \#023426), were crossed with either C57BI/6J mice (JAX mice, stock 000664) to generate hemizygote and wildtype Kiss1-Cre mice, or, with B6.Cg-Gt(ROSA) ${ }^{26 S o r t m 9(C A G-t d T o m a t o) H z e / J ~ f l o x e d-s t o p ~ r e p o r t e r ~ m i c e ~(J A X ~ m i c e, ~ s t o c k ~ \# 007907) ~ t o ~ g e n e r a t e ~}$ heterozygous Kiss1-Cre/tdTomato female mice. 


\section{Viral Vectors}

The adenoassociated virus (AAV) pAAV2/9.CAG.FLEX.GCaMP6s.WPRE.SV40 was

351 purchased from Addgene (Addgene viral prep \#100842-AAV9; http://n2t.net/addgene:100842;

352 RRID: Addgene_100842). Viral titers were reduced to $2.5 \times 10^{12}$ with $0.1 \mathrm{M}$ PBS before use.

353 Previous studies using cell-attached recordings of ARC kisspeptin neurons in acute brain slices

354 support that kisspeptin neuron activity is faithfully reported by changes in GCaMP6s fluorescence

355 following transfection with the current viral vector (22).

357 Surgical procedures

Stereotaxic viral injection and ovariectomy

Kiss1-Cre ${ }^{+/}$, Kiss1-Cre ${ }^{+/} /$tdTomato $^{+/-}$or Kiss1-Cre ${ }^{-/} /$tdTomato $^{+/-}$mice were anesthetized

360 with isoflurane (2\%) and placed in a stereotaxic frame (Stoelting Co. IL, USA). Using a Drill and

361 Microinjection Robot (Neurostar, Tubingen, Germany), a small hole was drilled into the skull $1 \mathrm{~mm}$

362 posterior to bregma and $0.92 \mathrm{~mm}$ lateral to midline. A 29 -gauge cannula attached to a $2.5 \mu \mathrm{L}$

363 Hamilton Syringe was loaded with 500nL of diluted AAV, angled at 6 degrees towards midline and

364 slowly lowered $5.88 \mathrm{~mm}$ ventral to dura into the unilateral ARC at a rate of $150 \mathrm{um} / \mathrm{min}$. The needle

365 was left in situ for $10 \mathrm{~min}$ before the viral vector was injected at a rate of $50 \mathrm{~nL} / \mathrm{min}$. Following

366 injection, syringes were left in situ for a further $10 \mathrm{~min}$ before the needle was slowly removed at a

367 rate of $150 \mathrm{um} / \mathrm{min}$. During the viral injection procedure, mice were bilaterally ovariectomized to

368 generate a state of absent steroid hormone inhibition of KNDy cell activity. Mice were returned to

369 clean home cages and pair housed until Kiss1-Cre mice were implanted with gradient reflective

370 index (GRIN) lenses 1-3 weeks after viral injection for calcium imaging. Kiss1-Cre/tdTomato mice

371 were transcardially perfused with $4 \%$ paraformaldehyde in $0.1 \mathrm{M}$ phosphate buffered saline (PBS) 
3723 weeks after viral injection for immunohistochemical analysis of viral specificity with Cre373 expressing arcuate kisspeptin cells.

379 designed for attachment of a miniaturized, single-photon fluorescent microscope (Inscopix Inc,

$380 \mathrm{nVoke}$ ). First, a $600 \mu \mathrm{m}$ diameter optical fiber (Thorlabs Inc, Cat: FT600UMT) with a sharpened tip 381 was inserted into polyimide tubing $(624 \mu \mathrm{m}$ diameter, cut to $8.4 \mathrm{~mm}$ length, Nordson Medical, Cat: 382 141-0159). The polyimide-optical fiber pairing was attached to the stereotaxic frame and implanted $383 \quad 200-300 \mu m$ above the arcuate nucleus. The implant was angled at 6 degrees towards midline to reduce motion artifacts from proximity to the third ventricle. The polyimide tubing was secured to

385 the skull using C\&B metabond dental cement (Parkell, Inc. Cat 375-0407) and, once dry, the optical 386 fiber was retracted. The integrated GRIN lens was inserted through the polyimide tubing until $387200 \mu m$ above the ARC and the baseplate was secured to the skull using C\&B dental cement. After 388 surgery, mice were housed individually and allowed to recover for at least 1 week before receiving 389 daily habituation for microscope attachment and serial blood sampling. No gross behavioral 390 abnormalities were observed in animals that received surgery.

\section{Habituation to microscope attachment and serial blood sampling}

One week following surgery, mice were habituated daily for 3-5 weeks to serial blood 394 sampling protocols as reported previously (50). Briefly, mice were trained to enter the hand of the 395 investigator from inside the home cage and the tail was stroked to mimic the blood collection protocol. Mice would be placed back in their home cage when samples were not being collected. 
397 In addition, mice were scruffed during handling for habituation to the restraint necessary for 398 microscope attachment and removal.

407 Tween20. Blood samples diluted in PBS-Tween20 were immediately frozen on dry ice and then 408 stored at $-20 \mathrm{C}$ until measurement of LH levels using ELISA.

\section{Post-processing of images}

All calcium imaging data were processed using IDPS software. First, data was cropped to

412 areas containing GCaMP6s fluorescence. Second, background was subtracted from the images 413 using a spatial band-passing Gaussian filter. Each video was corrected for motion using the mean

414 image of the entire video as a reference and the change in fluorescence (DF) relative to the resting $415 \mathrm{~F}(\mathrm{DF} / \mathrm{F})$. was generated. Within DF/F videos PCA-ICA was used to extract GCaMP6s fluorescent 416 responses associated with individual neurons (51). It should be noted that while the use of 417 automated segmentation programs to extract data from individual cells avoids bias and subjectivity 418 in cell identification for in vivo calcium imaging studies, it does not perfectly detect all fluorescent 419 cells within a recording. PCA-ICA data was manually inspected for signal from non-somatic 420 compartments, signal detected over multiple cells, or for cells with movement that is unable to be 421 corrected or distortion due to location on the edge of the imaging plane. Individual traces were 
422 exported for analysis in Excel and MATLAB (The Mathworks Inc.). Currently, there are two main 423 segmentation programs used for microendoscope data: PCA-ICA and constrained non-negative 424 matrix factorization for microendoscope imaging (CNMFe). Although CNMFe reduces the 425 contribution of fluorescent signal from cells above and below the imaging plane when compared to 426 PCA-ICA, it also has lower precision and identifies false positive cells (52). In this study, we found

427 CNMFe had poor recognition for the somatic compartment of cells (data not shown), and therefore 428 opted to use PCA-ICA data for analysis of calcium traces. Individual traces were exported for 429 analysis in Excel and MATLAB (The Mathworks Inc.).

432 Analysis of cell activation 
To assess the correlation of activity between individual cells, correlation matrix construction

448 was conducted using PRISM software. Z-Scored traces were separated into data points at baseline

449 activity and data points when fluorescence reached 3 times the standard deviation of the baseline

450 above the baseline. For both data sets, Pearson correlation was used to measure the correlation

451 coefficient between individual cells over the 60 -minute imaging period. $\mathrm{R}^{2}$ values from each cell-to-

452 cell correlation were averaged per animal. $\mathrm{R}^{2}$ values of zero indicate a random association between

453 neurons or no correlated activity, whereas values close to 1 or -1 indicate a high degree of positive

454 or negative correlation, respectively.

Order of cell activation

To determine whether KNDy neurons fire in a predictable pattern, the order that cell activity

rose above baseline (cells activated) and the order that cells reached peak amplitude was

459 determined for each episode recorded over a 60-minute period from each animal. From this data,

460 we calculated the percentage of the recorded cell population that displayed at least one instance

461 of activating for each order point over the 60-minute recording session. As the number of episodes

462 and cells recorded differed between animals, we normalized the number of cells and episodes.

463 Further, starting from the percentage of cells that displayed at least one instance of activating or

464 peaking first over 60 minutes of recording, we calculated the cumulative percentage of the 465 population when including cells that activated or peaked later during the episode.

469 ELISA, as previously reported (53). The assay sensitivity was $0.04 \mathrm{ng} / \mathrm{mL}$ and the intra- and inter470 assay coefficient of variation was $6.8 \%$ and $7.9 \%$, respectively. In serial blood samples from OVX 471 female mice, an LH pulse was identified when LH rose $>10 \%$ from the previous one or two samples 
472 and lowered $>20 \%$ in the following one or two samples, as previously described (22). The

473 percentage of LH pulses that were preceded by KNDy neuron activation and, conversely, the

474 percentage of KNDy population episodes followed by an LH pulse were calculated. Additionally, 475 the time from peak of $\mathrm{LH}$ to the peak of the preceding episode was measured. Finally, we measured 476 the time between peaks of adjacent synchronized episodes (Inter-SE-interval) and determined 477 whether LH pulsatile output occurred within the interval. From this, we calculated the percentage 478 of SE's that were followed by an LH pulse as determined by an ISI of over 5.5 minutes, between 4 479 and 5.5 minutes and under 4 minutes. with ice-cold 4\% PFA in 0.1M PBS (phosphate buffered saline). The brain was removed from the skull and the integrated GRIN lens with cannula was carefully removed. The brain was post-fixed 485 in $4 \%$ PFA for 1 hour at room temperature before switching to a $20 \%$ sucrose in $0.1 \mathrm{M}$ PBS solution 486 until sunk. Brains were then cut into 3 parallel series of coronal sections at $30 \mu \mathrm{m}$ thickness using 487 a freezing microtome. Tissue sections underwent 10 min washes in $0.1 \mathrm{M} \mathrm{PB}$ for 3 hours and were 488 mounted onto superfrost charged slides, air-dried and coverslipped using an aqueous mounting 489 medium in order to image endogenous fluorescence (Gelvotol; (54) containing the anti-fade agent 490 1,4-diazabicylo(2,2)octane (Sigma-Aldrich; $50 \mathrm{mg} / \mathrm{mL}$ ).

To assess the specificity of the viral vector with kisspeptin neurons, the colocalization of 492 GCaMP6s with tdTomato cells in Kiss1-Cre/tdTomato mice was calculated. Two sections 493 containing the rostral, middle and caudal arcuate nucleus were imaged per animal $(n=3)$ using an 494 Olympus FV3000 confocal microscope. A 20x objective was used to enable imaging of the entire 495 ARC area. Optical sections with a $1.25 \mu \mathrm{m}$ step size were acquired using $488 \mathrm{~nm}$ and $550 \mathrm{~nm}$ 496 channels. The number of GCaMP6s-positive cells, tdTomato cells, and dual labelled cells were 
497 quantified and the percentage of tdTomato-positive cells colocalized with GCaMP6s and the 498 percentage of GCaMP6s-positive cells colocalized with tdTomato was calculated.

499 To assess viral GCaMP expression and GRINS lens placement in Kiss1-Cre mice that 500 underwent in vivo $\mathrm{Ca}^{2+}$ imaging, tissue sections were imaged using epifluorescent microscopy 501 (DM500B, Lecia Microsystems) and a digital camera (Microfire A/R; Optronics) paired with 502 MicroBrightField Neurolucida Software (Williston, Vermont USA) and a 20x objective. Only mice 503 with accurate lens placement above the ARC with viral expression of GCAMP6s displayed visible 504 calcium signal and were included in this study. Approximately $50 \%$ of animals exhibited correct 505 lens placement, viral infection, and fluctuations in calcium signal, and these mice were used for 506 subsequent analysis $(n=5)$.

All statistical analyses were made using Prism8 (Graphpad Software Inc.) using the methods described below. All data, unless otherwise stated, is represented as the mean \pm SEM

511 per group ( $n=3$ animals in Figure 1, $n=5$ animals in Figs. 2-6) and a $p$ value of $<0.05$ was accepted 512 as statistically significant. First, statistical comparisons of $R^{2}$ values generated using Pearson's 513 correlation coefficient to determine the interaction between individual cells when at baseline activity 514 compared to above baseline were made using two-tailed unpaired Students T-Test (Figure 3). 515 Comparison of synchronized episodes in which $100 \%$ of cells were activated versus episodes 516 where less than $100 \%$ of cells were activated were conducted using two-tailed unpaired Students 517 T-Test (Figure 3). To compare the size of the recorded KNDy population that activated or peaked 518 in order of first to last, we compared data from each order point using two-way ANOVA with post519 hoc Bonferroni tests (Figures 4 C, D). The time between cell activation and peak amplitude for 520 leader cells that activated first, peaked first and non-leader cells was compared using one-way 521 ANOVA with Bonferroni post-test (Figure $4 \mathrm{G}$ ). Finally, the occurrence of LH pulsatile release 522 following episodes in which all cells versus some cells were active was compared using a two- 
523 tailed Students T-test (Figure 6 B), and LH pulse generation following SEs with different ISIs were

524 compared using one-way ANOVA with Bonferroni post-test (Figure $5 \mathrm{C}-\mathrm{E}$ ).

525

526

527

528

529

530

531

532

533

534

535

536

537

538

539

540

541

542

543

544

545

546

547

548

549

550

551

552

553

554

555

556

557

\section{Acknowledgments}

The authors would like to thank Dayanara B. Lohr for her excellent technical assistance during this research. Research reported in this publication was supported by the Eunice Kennedy Shriver National Institute of Child Health \& Human Development of the National Institutes of Health under Award Numbers K99HD096120 to A.M.M and R01HD039916 to M.N.L.

Author Contributions: A.M.M, L.M.C and M.N.L designed the research, A.M.M performed the research, A.M.M wrote the initial draft of the paper. All authors reviewed and edited the manuscript.

Competing Interest Statement: The authors declare that they have no competing interests.

\section{References}

1. Y. Nakai, T. Plant, D. Hess, E. Keogh, E. Knobil, On the sites of the negative and positive feedback actions of estradiol in the control of gonadotropin secretion in the rhesus monkey. Endocrinology 102, 1008-1014 (1978).

2. P. Belchetz, T. Plant, Y. Nakai, E. Keogh, E. Knobil, Hypophysial responses to continuous and intermittent delivery of hypopthalamic gonadotropin-releasing hormone. Science 202, 631-633 (1978).

3. N. de Roux et al., Hypogonadotropic hypogonadism due to loss of function of the KiSS1derived peptide receptor GPR54. Proceedings of the National Academy of Sciences 100, 10972-10976 (2003).

4. S. B. Seminara et al., The GPR54 gene as a regulator of puberty. New England Journal of Medicine 349, 1614-1627 (2003).

5. M. S. Irwig et al., Kisspeptin activation of gonadotropin releasing hormone neurons and regulation of KiSS-1 mRNA in the male rat. Neuroendocrinology 80, 264-272 (2004).

6. S. Messager et al., Kisspeptin directly stimulates gonadotropin-releasing hormone release via G protein-coupled receptor 54. Proceedings of the National Academy of Sciences of the United States of America 102, 1761-1766 (2005). 
558 7. R. L. Goodman et al., Kisspeptin neurons in the arcuate nucleus of the ewe express both dynorphin A and neurokinin B. Endocrinology 148, 5752-5760 (2007).

8. S. Ramaswamy et al., Neurokinin B stimulates GnRH release in the male monkey (Macaca mulatta) and is colocalized with kisspeptin in the arcuate nucleus. Endocrinology 151, 4494-4503 (2010).

9. V. M. Navarro et al., Regulation of gonadotropin-releasing hormone secretion by kisspeptin/dynorphin/neurokinin B neurons in the arcuate nucleus of the mouse. The Journal of neuroscience : the official journal of the Society for Neuroscience 29, 1185911866 (2009).

10. C. True, M. Kirigiti, P. Ciofi, K. L. Grove, M. S. Smith, Characterisation of arcuate nucleus kisspeptin/neurokinin B neuronal projections and regulation during lactation in the rat. Journal of neuroendocrinology 23, 52-64 (2011).

11. A. Hassaneen et al., Immunohistochemical characterization of the arcuate kisspeptin/neurokinin B/dynorphin (KNDy) and preoptic kisspeptin neuronal populations in the hypothalamus during the estrous cycle in heifers. The Journal of reproduction and development 62, 471-477 (2016).

12. Y. Wakabayashi et al., Neurokinin B and dynorphin A in kisspeptin neurons of the arcuate nucleus participate in generation of periodic oscillation of neural activity driving pulsatile gonadotropin-releasing hormone secretion in the goat. The Journal of neuroscience : the official journal of the Society for Neuroscience 30, 3124-3132 (2010).

13. A. M. Moore, L. M. Coolen, D. T. Porter, R. L. Goodman, M. N. Lehman, KNDy Cells Revisited. Endocrinology 159, 3219-3234 (2018).

14. M. C. Burke, P. A. Letts, S. J. Krajewski, N. E. Rance, Coexpression of dynorphin and neurokinin $B$ immunoreactivity in the rat hypothalamus: morphologic evidence of interrelated function within the arcuate nucleus. Journal of Comparative Neurology 498 , 712-726 (2006).

15. P. W. Weems et al., K-Opioid Receptor Is Colocalized in GnRH and KNDy Cells in the Female Ovine and Rat Brain. Endocrinology 157, 2367-2379 (2016).

16. C. D. Foradori, M. Amstalden, R. L. Goodman, M. N. Lehman, Colocalisation of dynorphin a and neurokinin $B$ immunoreactivity in the arcuate nucleus and median eminence of the sheep. Journal of neuroendocrinology 18, 534-541 (2006).

17. M. N. Lehman, L. M. Coolen, R. L. Goodman, Minireview: kisspeptin/neurokinin B/dynorphin (KNDy) cells of the arcuate nucleus: a central node in the control of gonadotropin-releasing hormone secretion. Endocrinology 151, 3479-3489 (2010).

18. A. E. Herbison, The Gonadotropin-Releasing Hormone Pulse Generator. Endocrinology 159, 3723-3736 (2018).

19. H. Okamura et al., Kisspeptin and GnRH pulse generation. Advances in experimental medicine and biology 784, 297-323 (2013).

20. H. Clarke, W. S. Dhillo, C. N. Jayasena, Comprehensive Review on Kisspeptin and Its Role in Reproductive Disorders. Endocrinology and metabolism (Seoul, Korea) 30, 124141 (2015).

21. R. L. Goodman et al., Kisspeptin, neurokinin B, and dynorphin act in the arcuate nucleus to control activity of the GnRH pulse generator in ewes. Endocrinology 154, 4259-4269 (2013).

22. J. Clarkson et al., Definition of the hypothalamic GnRH pulse generator in mice. Proceedings of the National Academy of Sciences of the United States of America 114, E10216-e10223 (2017).

23. S. Y. Han, T. McLennan, K. Czieselsky, A. E. Herbison, Selective optogenetic activation of arcuate kisspeptin neurons generates pulsatile luteinizing hormone secretion. Proceedings of the National Academy of Sciences 112, 13109-13114 (2015).

24. S. J. Krajewski, M. C. Burke, M. J. Anderson, N. T. McMullen, N. E. Rance, Forebrain projections of arcuate neurokinin $B$ neurons demonstrated by anterograde tract-tracing and monosodium glutamate lesions in the rat. Neuroscience 166, 680-697 (2010). 
611 25. J. Qiu et al., High-frequency stimulation-induced peptide release synchronizes arcuate

612

613

614

615

616

617

618

619

620

621

622

623

624

625

626

627

628

629

630

631

632

633

634

635

636

637

638

639

640

641

642

643

644

645

646

647

648

649

650

651

652

653

654

655

656

657

658

659

660

661

662

663

664

kisspeptin neurons and excites GnRH neurons. eLife 5, (2016).

26. K. Beale et al., The physiological role of arcuate kisspeptin neurons in the control of reproductive function in female rats. Endocrinology 155, 1091-1098 (2013).

27. M. A. Mittelman-Smith et al., Arcuate kisspeptin/neurokinin B/dynorphin (KNDy) neurons mediate the estrogen suppression of gonadotropin secretion and body weight. Endocrinology 153, 2800-2812 (2012).

28. M. A. Mittelman-Smith, S. J. Krajewski-Hall, N. T. McMullen, N. E. Rance, Ablation of KNDy Neurons Results in Hypogonadotropic Hypogonadism and Amplifies the SteroidInduced LH Surge in Female Rats. Endocrinology 157, 2015-2027 (2016).

29. M. Hu et al., Relative importance of the arcuate and anteroventral periventricular kisspeptin neurons in control of puberty and reproductive function in female rats. Endocrinology 156, 2619-2631 (2015).

30. T. Yamamura, Y. Wakabayashi, S. Ohkura, V. M. Navarro, H. Okamura, Effects of intravenous administration of neurokinin receptor subtype-selective agonists on gonadotropin-releasing hormone pulse generator activity and luteinizing hormone secretion in goats. The Journal of reproduction and development 61, 20-29 (2015).

31. S.-H. Yeo, A. E. Herbison, Projections of arcuate nucleus and rostral periventricular kisspeptin neurons in the adult female mouse brain. Endocrinology 152, 2387-2399 (2011).

32. J. Roa, M. Tena-Sempere, Connecting metabolism and reproduction: roles of central energy sensors and key molecular mediators. Molecular and cellular endocrinology 397, 4-14 (2014).

33. C. C. Nestor, M. J. Kelly, O. K. Ronnekleiv, Cross-talk between reproduction and energy homeostasis: central impact of estrogens, leptin and kisspeptin signaling. Hormone molecular biology and clinical investigation 17, 109-128 (2014).

34. P. Grachev et al., Neurokinin B signaling in the female rat: a novel link between stress and reproduction. Endocrinology 155, 2589-2601 (2014).

35. J. A. Yang et al., Acute Psychosocial Stress Inhibits LH Pulsatility and Kiss1 Neuronal Activation in Female Mice. Endocrinology 158, 3716-3723 (2017).

36. Y. Otsuki, K. Yamaji, M. Fujita, T. Takagi, O. Tanizawa, Serial plasma oxytocin levels during pregnancy and labor. Acta obstetricia et gynecologica Scandinavica 62, 15-18 (1983).

37. M. R. Perkinson, J. S. Kim, K. J. Iremonger, C. H. Brown, Visualising oxytocin neurone activity in vivo: The key to unlocking central regulation of parturition and lactation. Journal of neuroendocrinology, e13012 (2021).

38. V. Belin, F. Moos, Paired recordings from supraoptic and paraventricular oxytocin cells in suckled rats: recruitment and synchronization. The Journal of Physiology 377, 369-390 (1986).

39. V. Belin, F. Moos, P. Richard, Synchronization of oxytocin cells in the hypothalamic paraventricular and supraoptic nuclei in suckled rats: direct proof with paired extracellular recordings. Experimental brain research 57, 201-203 (1984).

40. H. J. McQuillan, S. Y. Han, I. Cheong, A. E. Herbison, GnRH pulse generator activity across the estrous cycle of female mice. Endocrinology 160, 1480-1491 (2019).

41. S. Y. Han, I. Cheong, T. McLennan, A. E. Herbison, Neural Determinants of Pulsatile Luteinizing Hormone Secretion in Male Mice. Endocrinology 161, (2020).

42. L. Wildt et al., Frequency and amplitude of gonadotropin-releasing hormone stimulation and gonadotropin secretion in the rhesus monkey. Endocrinology 109, 376-385 (1981).

43. R. P. Mclntosh, J. E. Mclntosh, Influence of the characteristics of pulses of gonadotrophin releasing hormone on the dynamics of luteinizing hormone release from perifused sheep pituitary cells. J Endocrinol 98, 411-421 (1983).

44. R. B. Perkins, J. E. Hall, K. A. Martin, Aetiology, previous menstrual function and patterns of neuro-endocrine disturbance as prognostic indicators in hypothalamic amenorrhoea. Human reproduction (Oxford, England) 16, 2198-2205 (2001). 
665 45. C. Coyle, R. E. Campbell, Pathological pulses in PCOS. Molecular and cellular

666

667

668

669

670

671

672

673

674

675

676

677

678

679

680

681

682

683

684

685

686

687

688

689

690

691

692

693

694 endocrinology 498, 110561 (2019).

46. J. T. George et al., Neurokinin B Receptor Antagonism in Women With Polycystic Ovary Syndrome: A Randomized, Placebo-Controlled Trial. The Journal of clinical endocrinology and metabolism 101, 4313-4321 (2016).

47. J. K. Prague et al., Neurokinin 3 receptor antagonism as a novel treatment for menopausal hot flushes: a phase 2 , randomised, double-blind, placebo-controlled trial. Lancet (London, England) 389, 1809-1820 (2017).

48. C. N. Jayasena et al., Subcutaneous injection of kisspeptin-54 acutely stimulates gonadotropin secretion in women with hypothalamic amenorrhea, but chronic administration causes tachyphylaxis. The Journal of Clinical Endocrinology \& Metabolism 94, 4315-4323 (2009).

49. R. M. Cravo et al., Characterization of Kiss1 neurons using transgenic mouse models. Neuroscience 173, 37-56 (2011).

50. R. B. McCosh, M. J. Kreisman, K. M. Breen, Frequent tail-tip blood sampling in mice for the assessment of pulsatile luteinizing hormone secretion. Journal of visualized experiments: JoVE, (2018).

51. E. A. Mukamel, A. Nimmerjahn, M. J. Schnitzer, Automated analysis of cellular signals from large-scale calcium imaging data. Neuron 63, 747-760 (2009).

52. A. M. Stamatakis et al., Miniature microscopes for manipulating and recording in vivo brain activity. Microscopy (Oxford, England), dfab028 (2021).

53. F. Steyn et al., Development of a methodology for and assessment of pulsatile luteinizing hormone secretion in juvenile and adult male mice. Endocrinology 154, 4939-4945 (2013).

54. L. B. Kuiper, L. N. Beloate, B. M. Dupuy, L. M. Coolen, Drug-taking in a socio-sexual context enhances vulnerability for addiction in male rats. Neuropsychopharmacology: official publication of the American College of Neuropsychopharmacology 44, 503-513 (2019). 
695

696

\section{Figures}

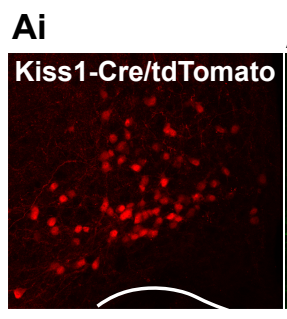

B

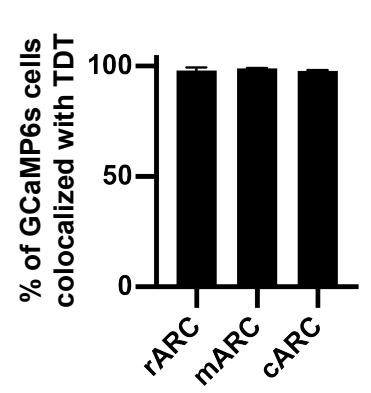

Aii

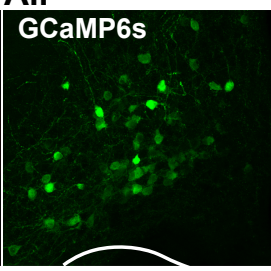

C

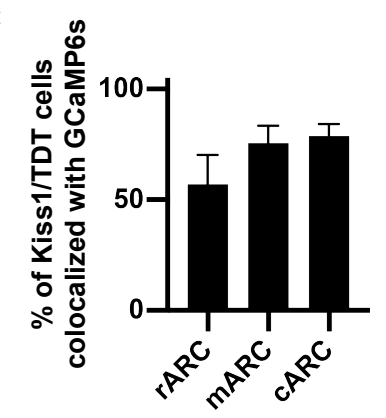

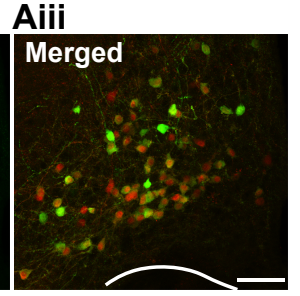

.

Figure 1. Viral delivery of the calcium indicator GCaMP6s is highly specific to arcuate kisspeptin neurons. A) Representative projected confocal images of the middle arcuate nucleus containing Kiss1-Cre cells expressing the tdTomato fluorophore (i, red ), cells transfected with a Cre-dependent viral vector containing the calcium indicator GCaMP6s (ii. green) and merged images illustrating the high degree of colocalization between kisspeptin cells and GCaMP6s (iii). B-C) Histograms depicting the percentage of GCaMP6-positive cells that are colocalized with Kiss1-Cre/tdTomato throughout the rostro-caudal extent of ARC: in the rostral $(r)$, middle $(m)$ and caudal (c) portions (B) and the percentage of tdTomato-expressing kisspeptin cells that express GCaMP6s. Scale bar $=100 \mu \mathrm{m}$. 


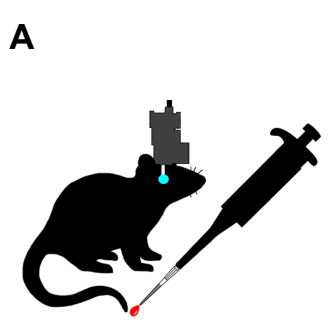

Di
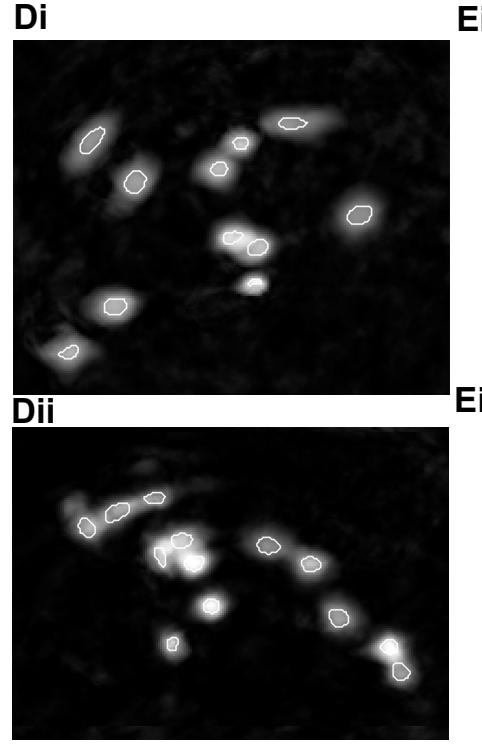

\section{Diii}

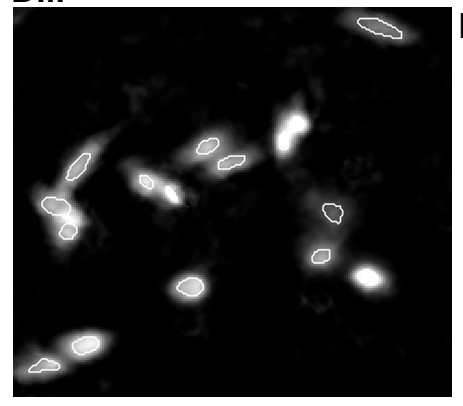

B

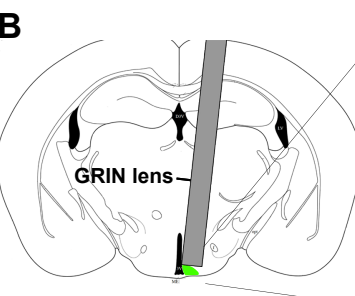

Ei
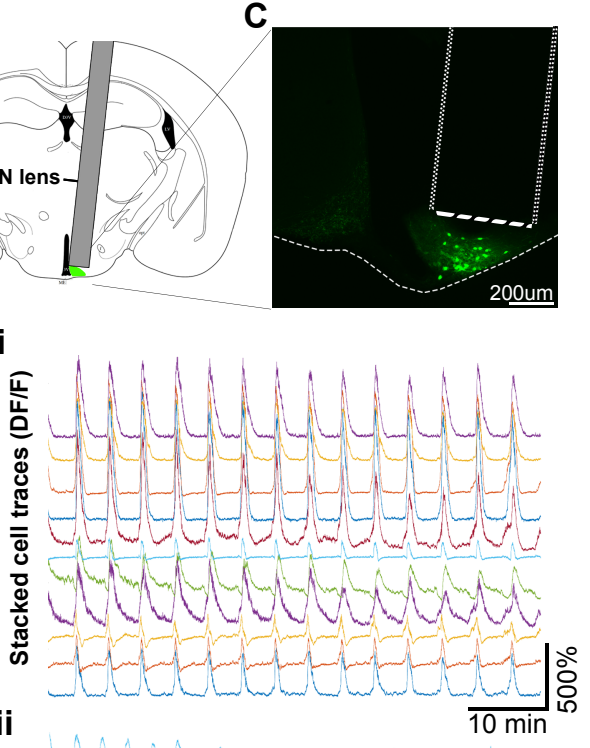

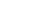

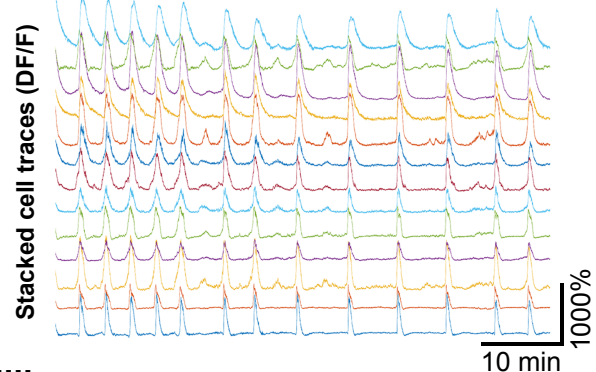

Eiii

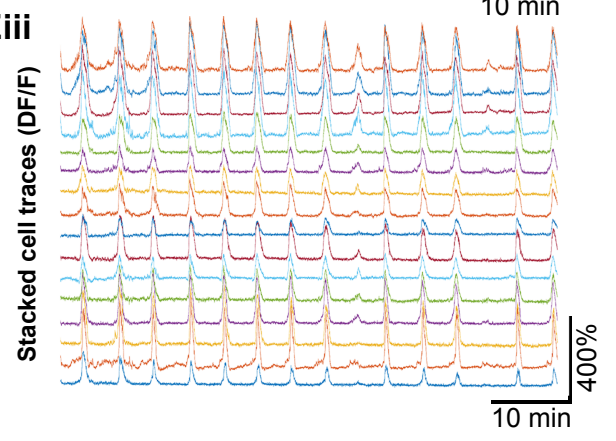

Figure 2. In vivo calcium imaging of KNDy cells in awake and freely moving mice reveals highly synchronized episodic activity at the single-cell level. A) Ovariectomized female Kiss1Cre mice with GCaMP6s expressed by ARC kisspeptin (KNDy) cells underwent 60 minutes of in vivo imaging of calcium activity in the arcuate nucleus using a microendoscope (Inscopix Inc). Regular blood samples were collected from the tail-tip for luteinizing hormone pulse analysis. Schematic representation (B) and fluorescent image (C) of GRIN lens placement above GCaMP6sexpressing KNDy cells. Di-iii) Images of KNDy cells from representative animals generated following segmentation by the principal component analysis and independent component (PCAICA) algorithm (white outlines). Ei-iii) Representative traces of calcium activity extracted from individual KNDy cells identified using PCA-ICA in Di-iii revealing synchronized and episodic changes in fluorescence (DF/F) in awake and freely moving mice. 


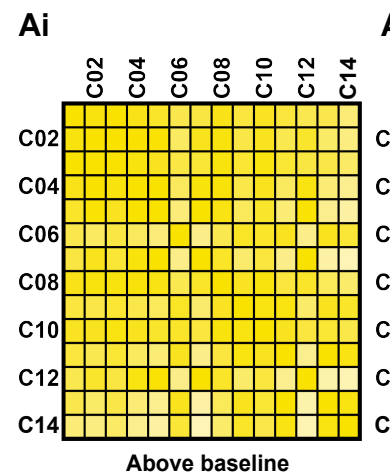

Aii

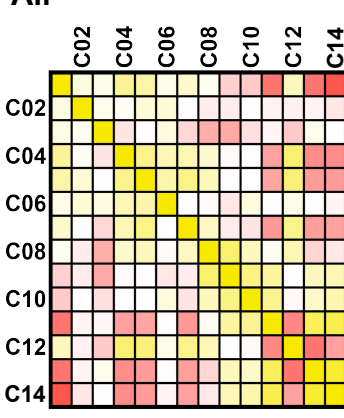

Baseline activity

E

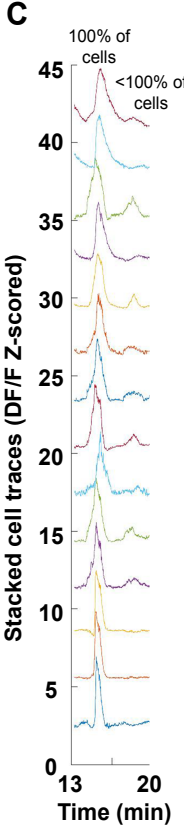

D

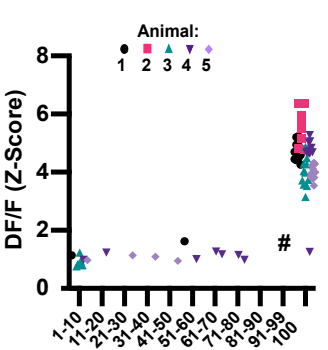

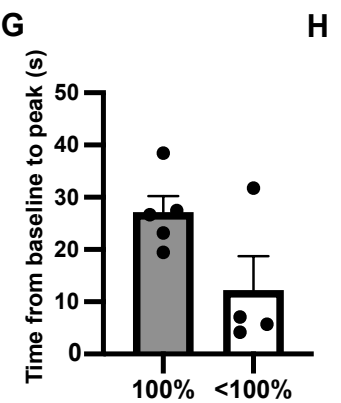

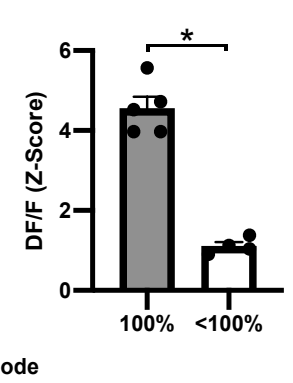

B

R2
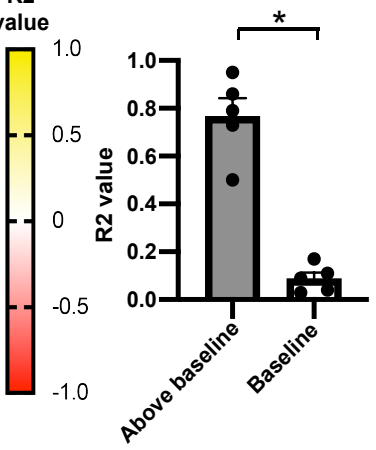

$\mathbf{F}$

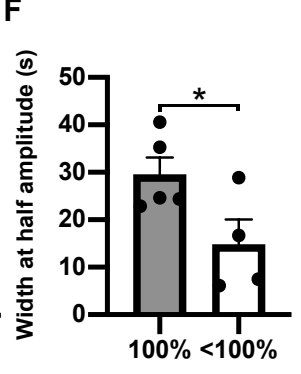

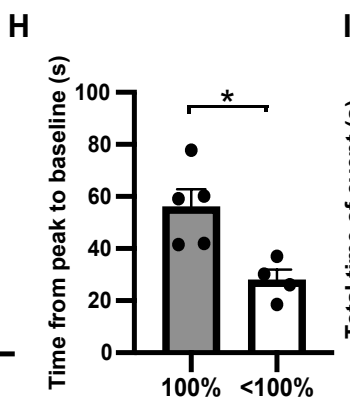

1

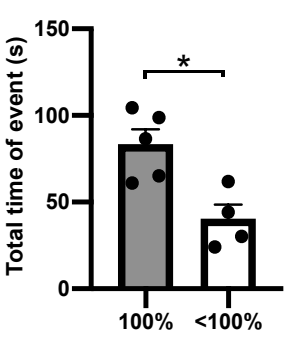

Figure 3. The KNDy neuron population displays either large, synchronized episodes of activation by all cells or smaller episodes of activation by subgroups of cells. A) Matrices for a representative mouse showing the correlation of activity $\left(R^{2}\right)$ between individual cells (C01-C14) when the average calcium activity for the population is above (i) or at (ii) baseline. B) The mean \pm SEM $R^{2}$ based on all animals $(n=5)$ is significantly higher when activity is elevated above baseline. C) Representative fluorescent traces from individual cells during a synchronized episode in which $100 \%$ of cells are robustly activated, followed by lower amplitude activity by less than $100 \%$ of cells. D) Graph demonstrating the amplitude of episodes (Z-scored DF/F) remains low unless $100 \%$ of cells are activated. Each data point represents an episode in each of the animals (74 episodes from 5 animals) E-I) Graphs demonstrating the mean \pm SEM $(n=5)$ amplitude of episodes per animal $(E)$ and the mean \pm SEM width of an episode at half amplitude $(F)$ is significantly higher when $100 \%$ of cells are activated compared to less than $100 \%$ of cells. The mean \pm SEM time from baseline to peak is not significantly dependent on the percentage of cells activated $(\mathrm{G})$, however the time from peak to baseline $(\mathrm{H})$ and the total time above baseline $(\mathrm{I})$ is significantly higher when $100 \%$ of cells are activated in an episode compared to less than $100 \% .{ }^{*}=p<0.05$. 
Ai

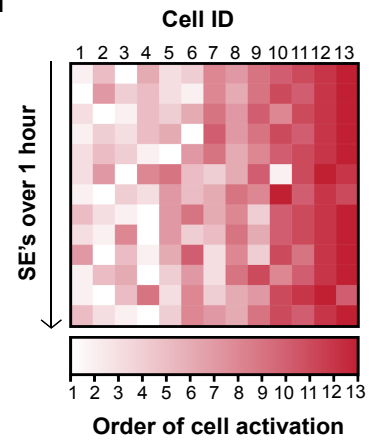

Aii

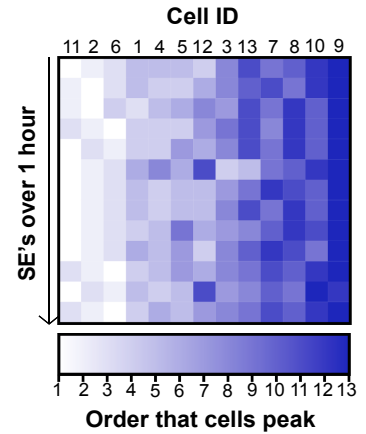

E

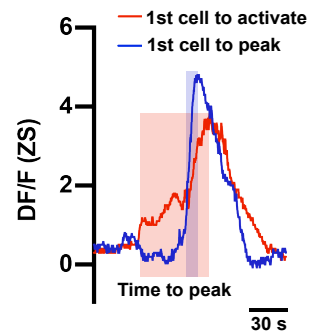

Bi

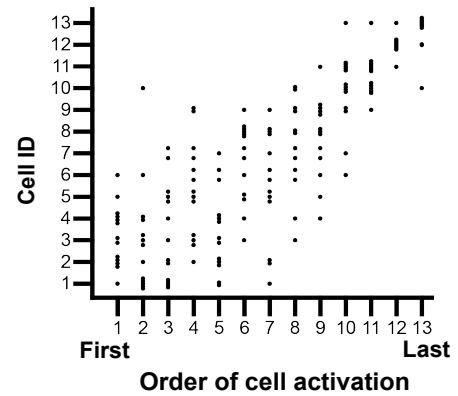

Bii

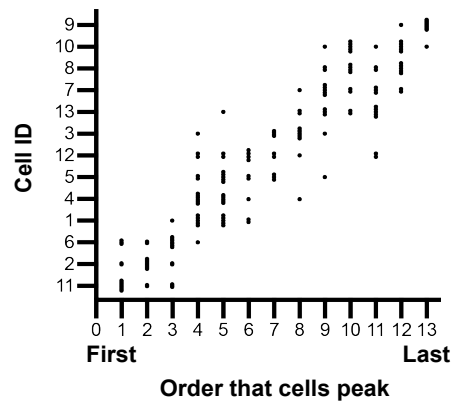

F

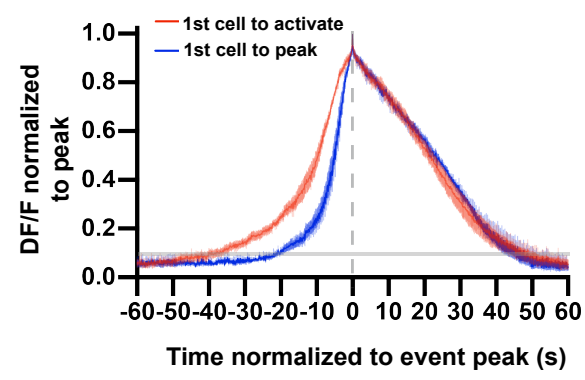

C

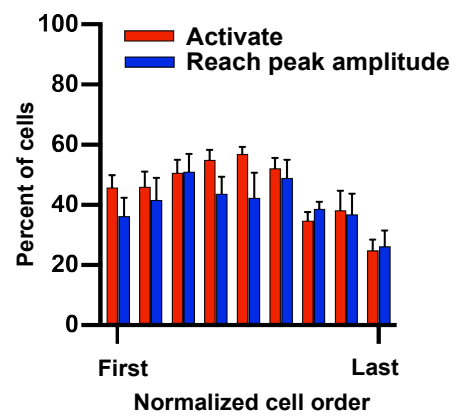

D

- Activate

- Reach peak amplitude

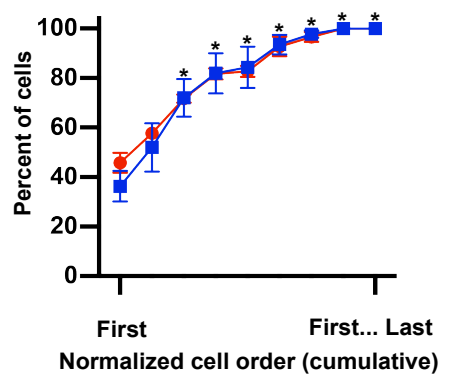

G

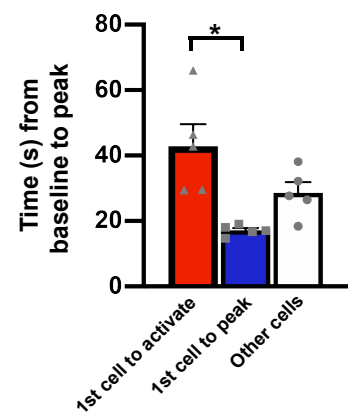

Fig 4. KNDy cells activate first and peak in a predictable temporal order during a synchronized episode. A) Heat-map from a representative animal depicting the order that fluorescence in individual cells (Cell ID, x-axis) elevates above baseline (i, activation) and reaches peak amplitude (ii) during synchronized episodes (y axis, 13 SE's in 60 minutes). The cell IDs in i correspond with the cell ID's in ii. B) Scatterplot mapping the order in which individual cells activate (i) and reach peak amplitude (ii) from the animal in A. C-D) Graphs demonstrating the mean \pm SEM percentage $(C)$ and the mean \pm SEM cumulative percentage (D) of KNDy cells per animal $(n=5)$ that activate or peak in order of first to last over multiple SEs in a 60-minute recording. ${ }^{*}$ in D indicates a significant increase compared to the percent of cells that activate or peak first. E) Calcium traces from representative cells that activate first (red) and peak first (blue) during a single synchronized episode. The shaded boxes depict the time from baseline to peak for the cell that activates first (red box) and the cell that peak first (blue box). F) The mean \pm SEM calcium fluorescence (normalized to peak) per animal $(n=5)$ of cells that activate first versus cells that peak first, graphed with time 0 as the peak of fluorescent traces (grey dotted line). The shaded line on the $y$ axis depicts the average point at which cell activity is above baseline. G) Graph illustrating the mean \pm SEM time from baseline to peak amplitude per animal $(n=5)$ for cells that activate first in an SE is significantly longer compared to cells that reach peak amplitude first. ${ }^{*}=p<0.05$. 

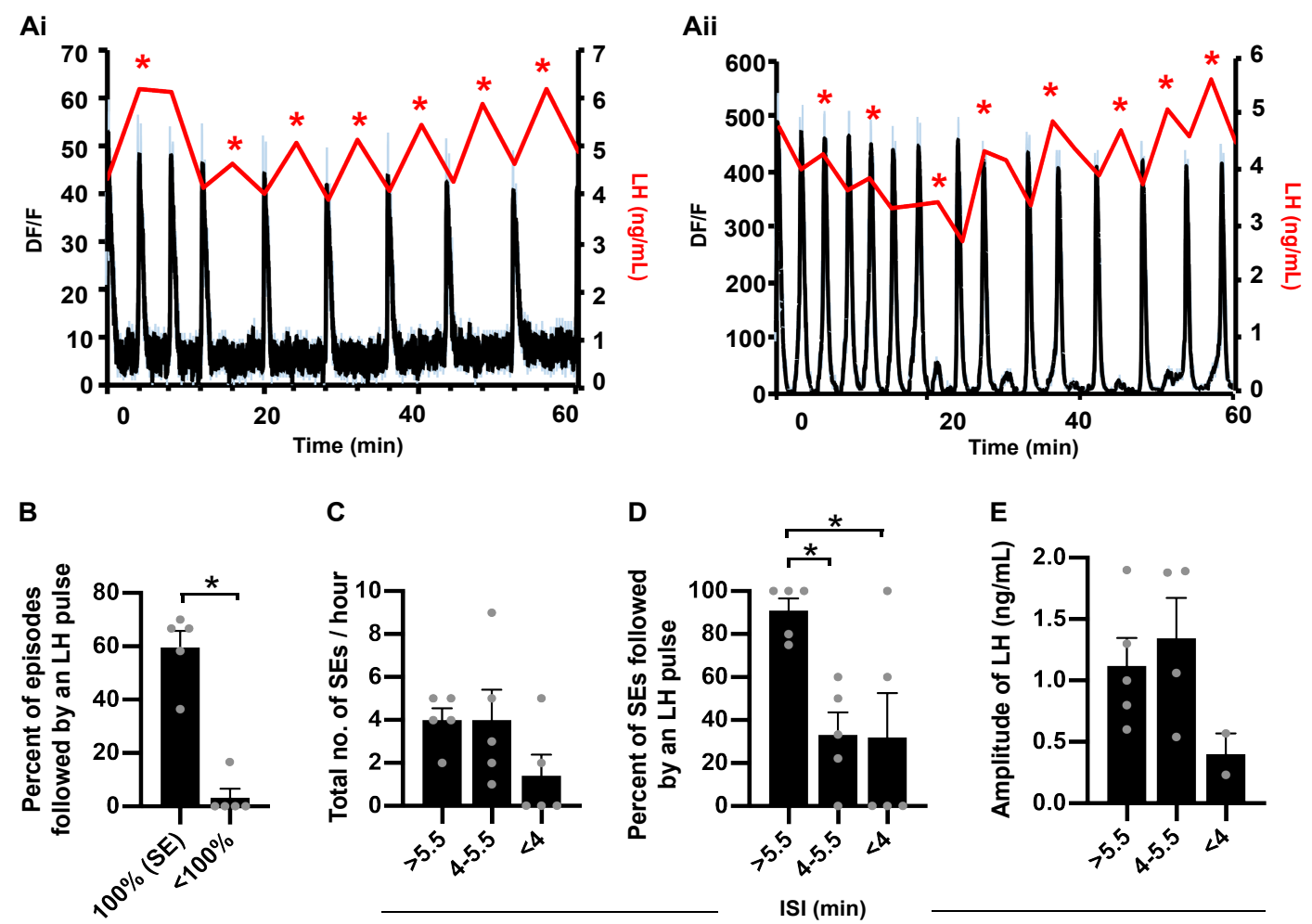

$\%$ of cells activated in an episode

$\mathrm{Fi}$

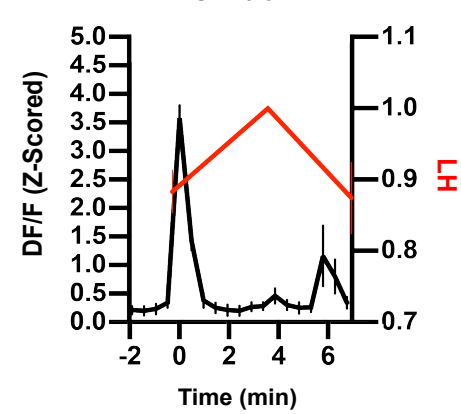

Fii

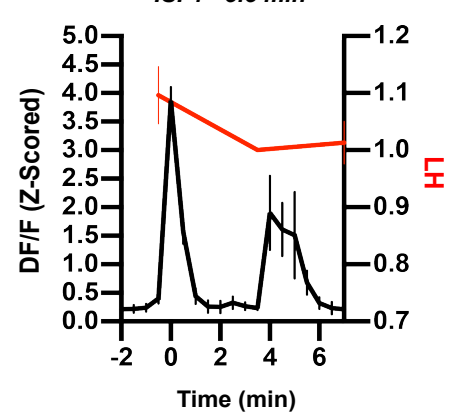

Fiii

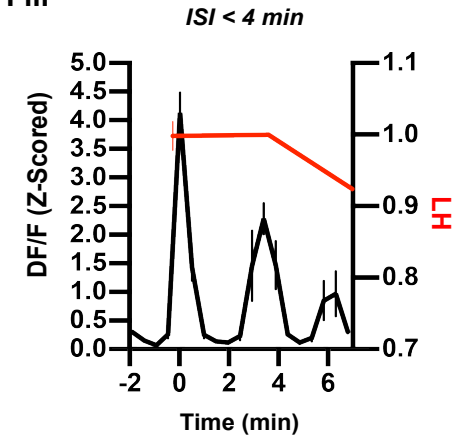

Fig 5. LH pulses are generated by synchronized KNDy population activity with an interval of over 5 minutes. Ai-ii) Representative examples from two animals of the mean (black line) \pm SEM (blue error bars) change in fluorescence (DF/F) within the KNDy neuron population over a 60minute recording coupled with $\mathrm{LH}$ pulsatile release (red line). ${ }^{*}$ depicts an LH pulse. B) The percentage of episodes with KNDy cell activity followed by an LH pulse reveal episodes with less than $100 \%$ of cells recruited rarely elicit an LH pulse. C) The number of SEs over a 60 -minute recording period that have an interval of over $5.5 \mathrm{~min}, 4-5.5 \mathrm{~min}$ and under $4 \mathrm{~min}$. D) Graph summarizing that synchronized episodes (SEs) with an inter-SE-interval (ISI) of over $5.5 \mathrm{~min}$ are followed by an LH pulse, whereas an ISI of under 5.5 minutes significantly reduces LH pulsatile release. E) Graph illustrating that the amplitude of $\mathrm{LH}$ release is lower when a pulse is generated following a KNDy SE with an ISI of less than 4 minutes. F) Fluorescent traces (peak at time 0) with an ISI of over $5.5 \mathrm{~min}{ }^{*}(\mathrm{i})$, between 4 to $5.5 \mathrm{~min}$ (ii) and under $4 \mathrm{~min}$ (iii) plotted against LH that has normalized to the sample collected after the KNDy SE peak. ${ }^{*} p<0.05$. Data in B-F are depicted as averages per animal and expressed as mean \pm SEM; $n=5 ;{ }^{*}=p<0.05$. 
$\mathbf{A i}$

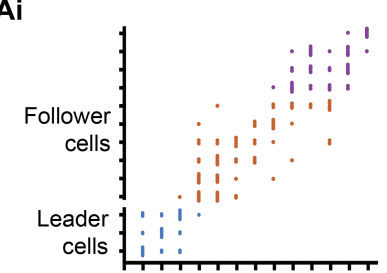

Aii

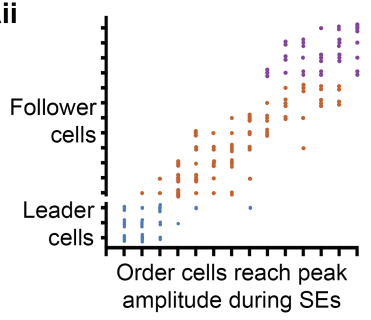

B

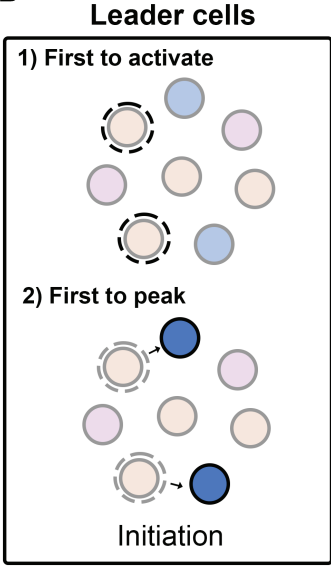

Follower cells

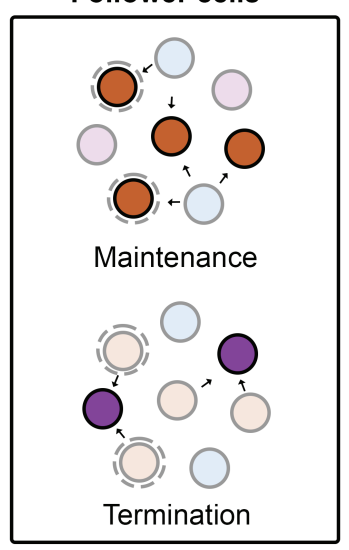

773 Fig 6. Predicted model for the temporal ordering of KNDy neuron activation during 774 synchronized episodes. A) Scatterplots mapping the order of cell activity during synchronized episodes (SE) from 60-minute recordings from two representative animals. Each dot represents the order in which a cell reached peak amplitude during a SE. KNDy neurons have been divided into "leader" and "follower cells" depending on their order of activation across multiple SEs and color-coded into the categories shown in B. B) Schematic depicting the predicted temporal activation of KNDy neurons that drives LH pulsatile release. First, a population of leader cells first initiate an LH pulse (1) by exhibiting an increase in activity towards threshold and driving activation of a subpopulation of leader cells that peak first (2, blue cells), which activates reciprocally connected follower cells. Follower cells are further divided into cells that peak during the maintenance (orange cells) and termination (purple cells) phase of the KNDy/GnRH/LH pulse. 